\title{
NUMERICAL SIMULATION OF UNSTEADY WATER-BASED NANOFLUID FLOW AND HEAT TRANSFER BETWEEN TWO ORTHOGONALLY MOVING POROUS COAXIAL DISKS
}

\author{
Kashif Ali, Muhammad Faroog Iqbal, Zubair Akbar, Muhammad Ashraf \\ Centre for Advanced Studies in Pure and Applied Mathematics, Bahauddin Zakariya University, Multan, Pakistan \\ e-mail: kashifali_381@yahoo.com
}

\begin{abstract}
We present the numerical study of unsteady hydromagnetic (MHD) flow and heat transfer characteristics of a viscous incompressible electrically conducting water-based nanofluid (containing $\mathrm{Al}_{2} \mathrm{O}_{3}$ nanoparticles) between two orthogonally moving porous coaxial disks with suction. Different from the classical shooting methodology, we employ a combination of a direct and an iterative method (SOR with optimal relaxation parameter) for solving the sparse systems of linear algebraic equations arising from the FD discretization of the linearized self similar nonlinear ODEs. Effects of the governing parameters on the flow and heat transfer are discussed and presented through tables and graphs. The findings of the present investigation may be beneficial for electronic industry in maintaining the electronic components under effective and safe operational conditions.
\end{abstract}

Keywords: porous disks, $\mathrm{Al}_{2} \mathrm{O}_{3}$ nanoparticles, viscous dissipation, wall expansion ratio, Joule heating

\section{Introduction}

The flow between two disks have many important applications in the fields of oceanography, biomechanics, rotating machinery, heat and mass exchangers, crystal growth processes, lubricants, computer storage devices and viscometries. In thrust bearings, the disks are separated by means of a lubricant injected through the disks. Furthermore, fluids with polymer additives have been used as improved lubricating oils in the modern lubrication technology (Connr et al., 1968).

In general, it is well known that conventional heat transfer fluids such as mineral oil, ethylene glycol and water have poor heat transfer properties as compared to those of most solids. To improve heat transfer, very small sized solid particles are added to the base fluid. The resulting new kind of the fluid (named as nanofluid) was firstly introduced by Choi (1995). The nanofluid is used to describe a mixture of a liquid and a solid. Such nanofluids have a very high thermal conductivity which is beyond the explanation of any existing theory. Nanofluids are also more stable and pose no additional problems such as sedimentation, additional pressure drop and erosion, due to the nano sized particles. The comprehensive references on nanofluids can be found in the recent book by Das and Choi (2007) and in the papers by Buongiorno (2006), Kakac and Pramuanjaroenkij (2009).

Injection or suction of a fluid through a porous bounding heated or cooled surface is of general interest in many practical applications including film cooling, control of the boundary layer etc. Fluid flow and convective heat transfer in rotor-stator systems (the rotor for a rotating disk and the stator for a stationary disk) are of great importance in turbomachinery and power engineering. Many researchers have worked on the problems related to disks with different wall conditions, for example, Usha and Ravindranthe (2001), Banchok et al. (2011), Elcrat (1976) and Rasmussen (1970). 
To our best knowledge no researcher has yet considered the behaviour of a nanofluid between two orthogonally moving porous disks. Therefore, in this paper, we consider the unsteady, laminar, incompressible, and two-dimensional flow of a nanofluid between two orthogonally moving porous coaxial disks, with an external magnetic field acting normally. We use a similarity transformation to reduce the governing partial differential equations to a set of nonlinear coupled ordinary differential equations in the dimensionless form, which are numerically solved by employing an algorithm based on the quasi-linearization and finite difference discretization. The ease in obtaining the numerical solution using this technique makes it superior than the shooting like approach used in our earlier investigations (Ashraf et al., 2009; Ashraf and Wehgal (2012). The effects of the governing parameters on the flow and heat transfer aspects of the problem are discussed.

\section{Mathematical formulation}

We consider hydromagnetic unsteady laminar viscous flow and heat transfer of an incompressible nanofluid (containing $\mathrm{Al}_{2} \mathrm{O}_{3}$ nanoparticles) between two orthogonally moving porous coaxial disks with suction in the presence of a transversally applied magnetic field. The induced magnetic field is assumed to be negligible as compared with the imposed field. The magnetic Reynolds number which is the ratio of the product of characteristic length and fluid velocity to the magnetic diffusivity is assumed to be small. It is used to compare the transport of magnetic lines of the force in a conducting fluid with the leakage of such lines from the fluid. For small magnetic Reynolds numbers, the magnetic field tends to relax towards a purely diffusive state. It is also assumed that there is no applied polarization and hence no electric field. The disks have the same permeability, move down or up uniformly at a time-dependent rate $a^{\prime}(t)$, and are distanced by $2 a(t)$.

The geometry of the problem suggests that the cylindrical coordinate system may be chosen with the origin at the middle of the two disks. With $u$ and $w$ being the velocity components in, respectively, the $r$ and $z$ direction, the governing equations for the problem, taking the viscous dissipation and Joule heating effects into account, are

$$
\begin{aligned}
& \frac{\partial u}{\partial r}+\frac{u}{r}+\frac{\partial w}{\partial z}=0 \\
& \frac{\partial u}{\partial t}+u \frac{\partial u}{\partial r}+w \frac{\partial u}{\partial z}=-\frac{1}{\rho_{n f}} \frac{\partial p}{\partial r}+v_{n f}\left(\frac{\partial^{2} u}{\partial r^{2}}+\frac{1}{r} \frac{\partial u}{\partial r}-\frac{u}{r^{2}}+\frac{\partial^{2} u}{\partial z^{2}}\right)-\frac{\sigma_{e} B_{0}^{2}}{\rho_{n f}} u \\
& \frac{\partial w}{\partial t}+u \frac{\partial w}{\partial r}+w \frac{\partial w}{\partial z}=-\frac{1}{\rho_{n f}} \frac{\partial p}{\partial z}+v_{n f}\left(\frac{\partial^{2} w}{\partial r^{2}}+\frac{1}{r} \frac{\partial w}{\partial r}+\frac{\partial^{2} w}{\partial z^{2}}\right) \\
& \frac{\partial T}{\partial t}+u \frac{\partial T}{\partial r}+w \frac{\partial T}{\partial z}=\alpha_{n f} \frac{\partial^{2} T}{\partial z^{2}}+\frac{\mu_{n f}}{\left(\rho c_{p}\right)_{n f}}\left(\frac{\partial u}{\partial z}\right)^{2}+\frac{1}{\left(\rho c_{p}\right)_{n f}} \sigma_{e} B_{0}^{2} u^{2}
\end{aligned}
$$

where $\sigma_{e}$ is the electrical conductivity, $B_{0}$ is the strength of the magnetic field, $p$ is the pressure, and $T$ is the temperature, $\alpha_{n f}$ is the thermal diffusivity, $\rho_{n f}$ is the density, $v_{n f}$ is the kinematics viscosity of the nanofluid, which are given by

$$
\begin{array}{ll}
v_{n f}=\frac{\mu_{n f}}{\rho_{n f}} \quad \mu_{n f}=\frac{\mu_{f}}{(1-\phi)^{2.5}} & \rho_{n f}=(1-\phi) \rho_{f}+\phi \rho_{s} \quad \alpha_{n f}=\frac{k_{n f}}{\left(\rho c_{p}\right)_{n f}} \\
\left(\rho c_{p}\right)_{n f}=(1-\phi)\left(\rho c_{p}\right)_{f}+\phi\left(\rho c_{p}\right)_{s} & \frac{k_{n f}}{k_{f}}=\frac{k_{s}+2 k_{f}-2 \phi\left(k_{f}-k_{s}\right)}{k_{s}+2 k_{f}+\phi\left(k_{f}-k_{s}\right)}
\end{array}
$$

where $\rho_{s}$ and $\rho_{f}$ are, respectively, the densities of the solid fractions and of the fluid, $\left(\rho c_{p}\right)_{n f}$ is the heat capacitance of the nanofluid, $k_{n f}$ is the effective thermal conductivity of the nanofluid, approximated by the Maxwell-Garnett model. 
The boundary conditions are

$$
\begin{array}{lll}
z=-a(t) & u=0 & w=-A a^{\prime}(t) \quad \text { and } \\
z=a(t) & u=0 & w=A a^{\prime}(t)
\end{array}
$$

Here $A$ is the measure of wall permeability, and the dash denotes the derivative w.r.t. time $t$.

After eliminating the pressure term from the governing equations, we introduce the following similarity transformation

$$
\eta=\frac{z}{a} \quad u=-\frac{r \nu_{f}}{a^{2}} F_{\eta}(\eta, t) \quad w=\frac{2 \nu_{f}}{a} F(\eta, t) \quad \theta=\frac{T-T_{2}}{T_{1}-T_{2}}
$$

The dimensions of $\nu_{f}$ are $\left[L^{2} T^{-1}\right]$, those of both $u$ and $w$ are $\left[L T^{-1}\right]$, and finally $[L]$ is the dimension of each $a$ and $r$, which when used in Eq. (2.4) give $F=a w /\left(2 \nu_{f}\right)$ and $F_{\eta}=-a^{2} u /\left(r \nu_{f}\right)$ as the two dimensionless velocities in the axial and radial direction, respectively. On the other hand, $\theta(\eta)$ being the ratio of two quantities having the same units, is also dimensionless.

The transformation given in Eq. (2.4) leads us to

$$
\begin{aligned}
& \frac{v_{n f}}{v_{f}} F_{\eta \eta \eta \eta}+\alpha\left(3 F_{\eta \eta}+\eta F_{\eta \eta \eta}\right)-2 F F_{\eta \eta \eta}-\frac{a^{2}}{v_{f}} F_{\eta \eta t}-\frac{\rho_{f}}{\rho_{n f}} M F_{\eta \eta}=0 \\
& \theta_{\eta \eta}-\frac{v_{f}}{\alpha_{n f}}(2 F-\eta \alpha) \theta_{\eta}+\left[(1-\phi)^{-2.5} F_{\eta \eta}^{2}+M F_{\eta}^{2}\right] E_{c} P_{r}\left(\frac{k_{f}}{k_{n f}}\right)-\frac{a^{2}}{\alpha_{n f}} \theta_{t}=0
\end{aligned}
$$

with boundary conditions

$$
\begin{array}{llll}
\eta=-1 & F=-R & F_{\eta}=0 & \text { and } \\
\eta=1 & F=R & F_{\eta}=0 &
\end{array}
$$

Here $T_{1}$ and $T_{2}$ (with $T_{1}>T_{2}$ ) are fixed temperatures of the lower and upper disks, respectively, $\alpha=a a^{\prime}(t) / v_{f}$ is the wall expansion ratio, $R=A a a^{\prime} /\left(2 v_{f}\right)$ is the permeability Reynolds number, $M=\sigma_{e} B_{0}^{2} a^{2} / \mu_{f}$ is the magnetic parameter, $P_{r}=\left(\mu c_{p}\right)_{f} / k_{f}$ is the Prandtl number, and $E_{c}=\left(r v_{f}\right)^{2} /\left[a^{4}\left(T_{1}-T_{2}\right)\left(c_{p}\right)_{f}\right]$ is the Eckert number.

It is worth to note that continuity Eq. $(2.1)_{1}$ is identically satisfied, which means that the proposed velocity is compatible with $(2.1)_{1}$ and, therefore, represents the possible fluid motion.

Finally, we set $f=F / R$, and consider the case (following Majdalani et al., 2002) when $\alpha$ is a constant, $f=f(\eta)$ and $\theta=\theta(\eta)$, which leads to $\theta_{t}=0$ and $f_{\eta \eta t}=0$. Thus we have

$$
\begin{aligned}
& \frac{v_{n f}}{v_{f}} f_{\eta \eta \eta \eta}+\alpha\left(3 f_{\eta \eta}+\eta f_{\eta \eta \eta}\right)-2 R f f_{\eta \eta \eta}-\frac{\rho_{f}}{\rho_{n f}} M f_{\eta \eta}=0 \\
& \theta_{\eta \eta}-\frac{v_{f}}{\alpha_{n f}}(2 R f-\eta \alpha) \theta_{\eta}+R^{2}\left[(1-\phi)^{-2.5} f_{\eta \eta}^{2}+M f_{\eta}^{2}\right] E_{c} P_{r}\left(\frac{k_{f}}{k_{n f}}\right)=0
\end{aligned}
$$

and

$$
\begin{array}{llll}
\eta=-1 & f=-1 & f_{\eta}=0 & \text { and } \\
\eta=1 & f=1 & f_{\eta}=0 &
\end{array}
$$

\section{Numerical solution}

An inspection of Eq. $(2.7)_{1}$ reveals that it can be readily integrated and becomes

$$
\frac{v_{n f}}{v_{f}} f_{\eta \eta \eta}+f_{\eta \eta}(\eta \alpha-2 R f)+f_{\eta}\left(2 \alpha+R f_{\eta}-\frac{\rho_{f}}{\rho_{n f}} M\right)=\beta
$$

where $\beta$ is the constant of integration to be determined. 
We consider this third order equation as the following system of coupled 1st and 2nd order ODEs

$$
f_{\eta}=q \quad \frac{v_{n f}}{v_{f}} q_{\eta \eta}+q_{\eta}(\eta \alpha-2 R f)+q\left(2 \alpha+R q-\frac{\rho_{f}}{\rho_{n f}} M\right)=\beta
$$

We solve the above equations along with Eqs. $(2.7)_{2}$, subject to the boundary conditions

$$
f( \pm 1)= \pm 1 \quad q( \pm 1)=0 \quad \theta(-1)=1 \quad \theta(1)=0
$$

For the numerical solution of the present problem, we first discretize Eqs. $(2.7)_{2}$ and $(3.2)_{2}$ at a typical grid point $\eta=\eta_{i}$ by employing central difference approximations for the derivatives. The resultant algebraic system is solved iteratively by the SOR method, subject to the appropriate boundary conditions given in Eq. (3.3). On the other hand, Eq. (3.2) 1 is integrated numerically by employing the 4th order Runge Kutta method, after every SOR iteration.

It is important to mention that the boundary condition $f(1)=1$ is not needed for the above mentioned computational procedure, and is used in finding the constant of integration $\beta$ which is the only unknown as compared to the three missing initial conditions in the usual shooting approach. In our earlier work, we used trial and error method to find $\beta$ but some one-dimensional zero finding algorithms may also be employed for the purpose. However, the sensitivity of $\beta$ with respect to the governing parameters makes it difficult to find it, and some manual effort is always required in every simulation to obtain its desired value. That is why we need some alternative approach which does not require finding any unknown and is entirely based on the FDM. In this paper, we discuss an alternative approach based on the quasi-linearization of the nonlinear ODEs.

\subsection{Quasi-linearization method}

In quasi-linearization, we construct two sequences of vectors $\left\{f^{(k)}\right\}$ and $\left\{\theta^{(k)}\right\}$, which converge to the numerical solutions to Eqs. $(2.7)_{1}$ and $(2.7)_{2}$, respectively. To construct $\left\{f^{(k)}\right\}$, we linearize Eq. (2.7) 1 by retaining only the first order terms, as follows.

We set

$$
\begin{aligned}
& G\left(f, f_{\eta}, f_{\eta \eta}, f_{\eta \eta \eta}, f_{\eta \eta \eta \eta}\right) \equiv \frac{v_{n f}}{v_{f}} f_{\eta \eta \eta \eta}+\alpha\left(3 f_{\eta \eta}+\eta f_{\eta \eta \eta}\right)-2 R f f_{\eta \eta \eta}-\frac{\rho_{f}}{\rho_{n f}} M f_{\eta \eta} \\
& G\left(f^{(k)}, f_{\eta}^{(k)}, f_{\eta \eta}^{(k)}, f_{\eta \eta \eta}^{(k)}, f_{\eta \eta \eta \eta}^{(k)}\right)+\left(f^{(k+1)}-f^{(k)}\right) \frac{\partial G}{\partial f^{(k)}}+\left(f_{\eta}^{(k+1)}-f_{\eta}^{(k)}\right) \frac{\partial G}{\partial f_{\eta}^{(k)}} \\
& \quad+\left(f_{\eta \eta}^{(k+1)}-f_{\eta \eta}^{(k)}\right) \frac{\partial G}{\partial f_{\eta \eta}^{(k)}}+\left(f_{\eta \eta \eta}^{(k+1)}-f_{\eta \eta \eta}^{(k)}\right) \frac{\partial G}{\partial f_{\eta \eta \eta}^{(k)}}+\left(f_{\eta \eta \eta \eta}^{(k+1)}-f_{\eta \eta \eta \eta}^{(k)}\right) \frac{\partial G}{\partial f_{\eta \eta \eta \eta}^{(k)}}=0 \\
& \frac{v_{n f}}{v_{f}} f_{\eta \eta \eta \eta}^{(k+1)}+\left(\eta \alpha-2 R f^{(k)}\right) f_{\eta \eta \eta}^{(k+1)}+\left(3 \alpha-\frac{\rho_{f}}{\rho_{n f}} M\right) f_{\eta \eta}^{(k+1)}-2 R f_{\eta \eta \eta}^{(k)} f^{(k+1)}=-2 R f_{\eta \eta \eta}^{(k)} f^{(k)}
\end{aligned}
$$

Now Eqs. (3.4) gives a system of linear differential equations, with $f^{k}$ being the numerical solution vector of the $k$-th equation. To solve the linear ODEs, we replace the derivatives with their central difference approximations, giving rise to the sequence $\left\{f^{(k)}\right\}$ generated by the following linear system

$$
A f^{(k+1)}=B \quad \text { with } \quad A \equiv A\left(f^{(k)}\right) \quad \text { and } \quad B \equiv B\left(f^{(k)}\right)
$$

where $n$ is the number of grid points. The matrices $A_{n \times n}$ and $B_{n \times 1}$ are initialized as follows 


$$
\left.\begin{array}{l}
A_{1,1}=1 \quad B_{1}=-1 \\
A_{2,1}=-8 d_{1}+2 h\left(\alpha \eta_{2}-2 R f_{2}\right)+2\left(3 \alpha-M d_{2}\right) h^{2} \\
A_{2,2}=14 d_{1}-4\left(3 \alpha-M d_{2}\right) h^{2}-2 h R\left(f_{4}-2 f_{3}+2 f_{1}-f_{2}\right)-h\left(\alpha \eta_{2}-2 R f_{2}\right) \\
A_{2,3}=-8 d_{1}-2 h\left(\alpha \eta_{2}-2 R f_{2}\right)+2\left(3 \alpha-M d_{2}\right) h^{2} \\
A_{2,4}=2 d_{1}+h\left(\alpha \eta_{2}-2 R f_{2}\right) \\
B_{2}=-2 h R f_{2}\left(-f_{2}+2 f_{1}-2 f_{3}+f_{4}\right) \\
A_{i, i-2}=2 d_{1}-h\left(\alpha \eta_{i}-2 R f_{i}\right) \\
A_{i, i-1}=-8 d_{1}+2 h\left(\alpha \eta_{i}-2 R f_{i}\right)+2\left(3 \alpha-M d_{2}\right) h^{2} \\
A_{i, i}=12 d_{1}-4\left(3 \alpha-M d_{2}\right) h^{2}-2 h R\left(f_{i+2}-2 f_{i+1}+2 f_{i-1}-f_{i-2}\right) \\
A_{i, i+1}=-8 d_{1}-2 h\left(\alpha \eta_{i}-2 R f_{i}\right)+2\left(3 \alpha-M d_{2}\right) h^{2} \\
A_{i, i+2}=2 d_{1}+h\left(\alpha \eta_{i}-2 R f_{i}\right) \\
B_{i}=-2 h R f_{i}\left(f_{i+2}-2 f_{i+1}+2 f_{i-1}-f_{i-2}\right) \\
A_{n-1, n-3}=2 d_{1}-h\left(\alpha \eta_{n-1}-2 R f_{n-1}\right) \\
A_{n-1, n-2}=-8 d_{1}+2 h\left(\alpha \eta_{n-1}-2 R f_{n-1}\right)+2\left(3 \alpha-M d_{2}\right) h^{2} \\
A_{n-1, n-1}=14 d_{1}-4\left(3 \alpha-M d_{2}\right) h^{2}-2 h R\left(f_{n-1}-2 f_{n}+2 f_{n-2}-f_{n-3}\right) \\
\quad+h\left(\alpha \eta_{n-1}-2 R f_{n-1}\right) \\
\begin{array}{l}
A_{n-1, n}=-8 d_{1}-2 h\left(\alpha \eta_{n-1}-2 R f_{n-1}\right)+2\left(3 \alpha-M d_{2}\right) h^{2} \\
B_{n-1}=-2 h R f_{n-1}\left(f_{n-1}-2 f_{n}+2 f_{n-2}-f_{n-3}\right) \\
A_{n, n}=1
\end{array} B_{n}=1 . \\
\quad
\end{array}\right\}
$$

where

$$
h=\frac{2}{n-1} \quad d_{1}=\frac{(1-\phi)^{-2.5}}{1-\phi+\phi \frac{\rho_{s}}{\rho_{f}}} \quad d_{2}=\frac{1}{1-\phi+\phi \frac{\rho_{s}}{\rho_{f}}}
$$

(The superscripts have been dropped for simplicity and clarity)

On the other hand, Eq. $(2.7)_{2}$ is linear in $\theta$, and therefore, in order to generate the sequence $\left\{\theta^{(k)}\right\}$, it may be written as

$$
\theta_{\eta \eta}^{(k+1)}-\frac{v_{f}}{\alpha_{n f}}\left(2 R f^{(k+1)}-\eta \alpha\right) \theta_{\eta}^{(k+1)}+R^{2}\left[(1-\phi)^{-2.5} f_{\eta \eta}^{(k+1)^{2}}+M f_{\eta}^{(k+1)^{2}}\right] E_{c} P_{r}\left(\frac{k_{f}}{k_{n f}}\right)=0
$$

Importantly, $f^{(k+1)}$ is considered to be known in the above equation and its derivatives are approximated by the central differences. We outline the computational procedure as follows:

- Provide the initial guess $f^{(0)}$ and $\theta^{(0)}$, satisfying the boundary conditions given in Eq. (2.8)

- Solve the linear system given by Eq. (3.5) to find $f^{(1)}$

- Use $f^{(1)}$ to solve the linear system arising from the FD discritization of Eq. (3.8) to get $\theta^{(1)}$

- Take $f^{(1)}$ and $\theta^{(1)}$ as the new initial guesses and repeat the procedure to generate the sequences $\left\{f^{(k)}\right\}$ and $\left\{\theta^{(k)}\right\}$ which, respectively, converge to $f$ and $\theta$ (the numerical solutions of Eqs. $(2.7)_{1}$ and $\left.(2.7)_{2}\right)$

- The two sequences are generated until $\max \left\{\left\|f^{(k+1)}-f^{(k)}\right\|_{L_{\infty}},\left\|\theta^{(k+1)}-\theta^{(k)}\right\|_{L_{\infty}}\right\}<10^{-6}$.

It is important to note that the matrix $A$ in Eqs. (3.6) is pentadiagonal and not diagonally dominant, and hence the iterative method (like SOR) may fail or work very poorly. Therefore, some direct method like the LU factorization or Guassian elimination with full pivoting (to ensure stability) may be employed. 
On the other hand, Eq. (3.8) gives rise to the diagonally dominant algebraic system when discretized using the central differences. Therefore, we are in position to use the SOR method for this equation. In this case, we also optimize the relaxation parameter $\omega$ by following Nakamura (1991). Instead of giving the details, we just outline the optimization procedure as follows:

- The SOR procedure is started with an initial guess for the value of the relaxation parameter $\omega$ to iteratively solve the linear system on some suitable basic grid.

- After each relaxation sweep except the first one, the quantities given by

$$
\mu_{\omega}^{(t)}=\sqrt{\frac{N^{(t)}}{N^{(t-1)}}} \quad \mu_{J}^{(t)}=\frac{\mu_{\omega}^{(t)}+\omega-1}{\omega \sqrt{\mu_{\omega}^{(t)}}}
$$

are computed, where

$$
N^{(t)}=\sum_{i}\left(U_{i}^{(t)}-U_{i}^{(t-1)}\right)^{2}
$$

Note that the upper index $t$ stands for the relaxation sweep and the lower index $i$ represents the grid point.

- The optimal relaxation parameter is then estimated from

$$
\omega_{o p t}^{(t)}=\frac{2}{1+\sqrt{1-\left(\mu_{J}^{(t)}\right)^{2}}}
$$

- The process of optimization is stopped once the criterion

$$
\left|\mu_{\omega}^{(t)}-\mu_{\omega}^{(t-1)}\right|<\mathrm{TOL}_{\omega_{o p t}}
$$

is satisfied subject to $t>1$ and $\mu_{\omega}^{(t)}<1$. The iterative process is continued with $\omega=\omega_{\text {opt }}$ until convergence is achieved. $\mathrm{TOL}_{\omega_{\text {opt }}}$ is chosen to lie in the range $10^{-5} \leqslant \mathrm{TOL}_{\omega_{\text {opt }}} \leqslant 10^{-3}$ depending upon the density of the grid points. For a coarse grid, larger values result in a rapid convergence, while for a fine grid, smaller values may be chosen for better estimation of $\omega_{\text {opt }}$.

We may improve the order of accuracy of the solution by solving the problem again on the grid with a step $h / 2$ and $h / 4$, and then using the Richardson extrapolation, which have been carried out at not only the common grid points but also at the skipped points, by following Roache and Knupp (1993). Any extrapolation scheme (Deuflhard, 1983) may be used for this purpose, but we have used the polynomial extrapolation.

Moreover, a good initial guess for the solution on the finer grid can be obtained by injecting the previous coarse grid solution. Many operators may be found for this purpose, but we have used the following linear operator which is simpler and easier to implement

$$
W_{2 i-1} \leftarrow U_{i}^{c} \quad W_{2 i} \leftarrow \frac{1}{2}\left(U_{i}^{c}+U_{i+1}^{c}\right)
$$

where $U^{c}$ represents the coarse grid solution and $W$ is the required initial guess.

\section{Results and discussion}

The physical quantities of our interest are the shear stresses and the heat transfer rates at the disks which are, respectively, proportional to $f^{\prime \prime}(-1)$ and $\theta^{\prime}(-1)$. Due to symmetry of the problem, the results are given only at the lower disk. The parameters for the present study 
are the Reynolds number $R$, the magnetic parameter $M$, the nanoparticle volume fraction parameter $\phi$, the wall expansion ratio $\alpha$ and the Eckert number $E c$. It is worth to note that $\alpha<0$ or $\alpha>0$ according to the case when the disks are approaching each other or moving away, whereas $R<0$ for suction. In the present study, we need to consider the effects of the parameters $R, M, \phi, \alpha, E c$ on the following quantities:

- Axial and radial velocity profiles

- Temperature distribution

- Shear stress and heat transfer rate at the disk.

As we are considering the water-based nanofluid containing $\mathrm{Al}_{2} \mathrm{O}_{3}$ nanoparticles, we take the following values for the study: $\rho_{f}=997.1, \rho_{s}=3970, k_{s}=40, k_{f}=0.613,\left(c_{p}\right)_{s}=765$, $\left(c_{p}\right)_{f}=4179$ and $\operatorname{Pr}=6.2$. It is to be noted that the case $\phi=0$ corresponds to pure water.

Table 1 shows the convergence of our numerical results as the step-size decreases, which gives us confidence on our computational procedure. It may be shown, as in our previous work (Ali et al., 2013), that the shear stress and the heat transfer rate at each disk are proportional to the values of $f^{\prime \prime}$ and $\theta^{\prime}$, respectively, at $\eta=-1$. Therefore, in the Tables 1-6, we have considered the effects of the governing parameters on $f^{\prime \prime}(-1)$ and $\theta^{\prime}(-1)$. It is clear from Table 2 that the external magnetic field increases the shear stress as well as the heat transfer rate, whether the disks are approaching each other or moving away. Addition of nanoprticles to water results in remarkably increasing the heat transfer rate while decreasing the shear stress for the two cases of $\alpha$ as predicted by Table 3. On the other hand, suction increases both the shear stress and the heat transfer rate when the disks are moving away, but decreases the shear stress while increasing the heat transfer rate for the other case (clear from Table 4). Table 5 predicts that both the shear stress and heat transfer rate increase when the disks are approaching each other, whereas the opposite trend is noted if the disks are moving away. Table 6 shows that the viscous dissipation always increases the heat transfer rate at the disks.

Table 1. Dimensionless velocity $f(\eta)$ on three grid sizes and extrapolated values for $R=-10$, $\phi=0.3, \alpha=-5$ and $M=1$

\begin{tabular}{|c|c|c|c|c|}
\hline \multirow{2}{*}{$\eta$} & \multicolumn{4}{|c|}{$f(\eta)$} \\
\cline { 2 - 5 } & $\begin{array}{c}\text { 1st grid } \\
(h=0.02)\end{array}$ & $\begin{array}{c}\text { 2nd grid } \\
(h=0.01)\end{array}$ & $\begin{array}{c}\text { 3rd grid } \\
(h=0.005)\end{array}$ & $\begin{array}{c}\text { Extrapolated } \\
\text { values }\end{array}$ \\
\hline \hline 0 & 0 & 0 & 0 & 0 \\
\hline 0.2 & 0.3054554 & 0.3054592 & 0.3054602 & 0.3054605 \\
\hline 0.4 & 0.5806770 & 0.5806883 & 0.5806911 & 0.5806920 \\
\hline 0.6 & 0.8011234 & 0.8011416 & 0.8011461 & 0.8011476 \\
\hline 0.8 & 0.9470534 & 0.9470702 & 0.9470744 & 0.9470758 \\
\hline
\end{tabular}

Table 2. Effect of the magnetic parameter $M$ on $f^{\prime \prime}(-1)$ and $\theta^{\prime}(-1)$ for $\phi=0.2, R=-10$ and $E c=0.1$

\begin{tabular}{|c|c|c|c|c|}
\hline \multirow{2}{*}{$M$} & \multicolumn{2}{|c|}{$\alpha=2$} & \multicolumn{2}{c|}{$\alpha=-2$} \\
\cline { 2 - 5 } & $f^{\prime \prime}(-1)$ & $\theta^{\prime}(-1)$ & $f^{\prime \prime}(-1)$ & $\theta^{\prime}(-1)$ \\
\hline \hline 0 & 1.8580 & 2.9180 & 2.3175 & 5.4619 \\
\hline 2 & 1.9009 & 3.0519 & 2.3685 & 5.6988 \\
\hline 4 & 1.9439 & 3.1893 & 2.4194 & 5.9406 \\
\hline 6 & 1.9871 & 3.3300 & 2.4704 & 6.1873 \\
\hline 8 & 2.0303 & 3.4740 & 2.5213 & 6.4388 \\
\hline
\end{tabular}


Table 3. Effect of the nanoparticle volume fraction parameter $\phi$ on $f^{\prime \prime}(-1)$ and $\theta^{\prime}(-1)$ for $M=2, R=-10$ and $E c=0.1$

\begin{tabular}{|c|c|c|c|c|}
\hline \multirow{2}{*}{$\phi$} & \multicolumn{2}{|c|}{$\alpha=2$} & \multicolumn{2}{c|}{$\alpha=-2$} \\
\cline { 2 - 5 } & $f^{\prime \prime}(-1)$ & $\theta^{\prime}(-1)$ & $f^{\prime \prime}(-1)$ & $\theta^{\prime}(-1)$ \\
\hline \hline 0 & 1.9200 & 1.6794 & 2.3936 & 3.1664 \\
\hline 0.05 & 1.9103 & 1.9174 & 2.3823 & 3.6112 \\
\hline 0.1 & 1.9044 & 2.2135 & 2.3749 & 4.1606 \\
\hline 0.15 & 1.9013 & 2.5839 & 2.3704 & 4.8430 \\
\hline 0.2 & 1.9009 & 3.0519 & 2.3685 & 5.6988 \\
\hline
\end{tabular}

Table 4. Effect of the permeability Reynolds number $R$ on $f^{\prime \prime}(-1)$ and $\theta^{\prime}(-1)$ for and $E c=0.1$

\begin{tabular}{|c|c|c|c|c|}
\hline \multirow{2}{*}{$R$} & \multicolumn{2}{|c|}{$\alpha=2$} & \multicolumn{2}{c|}{$\alpha=-2$} \\
\cline { 2 - 5 } & $f^{\prime \prime}(-1)$ & $\theta^{\prime}(-1)$ & $f^{\prime \prime}(-1)$ & $\theta^{\prime}(-1)$ \\
\hline \hline-2 & 1.7950 & 0.4395 & 3.5007 & 3.0065 \\
\hline-4 & 1.8234 & 1.0183 & 2.9193 & 3.8376 \\
\hline-6 & 1.8573 & 1.6661 & 2.6265 & 4.4320 \\
\hline-8 & 1.8826 & 2.3498 & 2.4660 & 5.0472 \\
\hline-10 & 1.9009 & 3.0519 & 2.3685 & 5.6988 \\
\hline
\end{tabular}

Table 5. Effect of the wall expansion ratio $\alpha$ on $f^{\prime \prime}(-1)$ and $\theta^{\prime}(-1)$ for $\phi=0.2, R=-10$, $M=2$ and $E c=0.1$

\begin{tabular}{|c|c|c|}
\hline$\alpha$ & $f^{\prime \prime}(-1)$ & $\theta^{\prime}(-1)$ \\
\hline \hline-5 & 2.8351 & 9.5692 \\
\hline-2 & 2.3685 & 5.6988 \\
\hline-1 & 2.2370 & 4.8414 \\
\hline 1 & 2.0041 & 3.5434 \\
\hline 2 & 1.9009 & 3.0519 \\
\hline 5 & 1.6355 & 1.9997 \\
\hline
\end{tabular}

Table 6. Effect of the Eckert number $E c$ on $\theta^{\prime}(-1)$ for $R=-10, \phi=0.2$ and $M=2$

\begin{tabular}{|c|c|c|}
\hline \multirow{2}{*}{$E c$} & \multicolumn{2}{|c|}{$\theta^{\prime}(-1)$} \\
\cline { 2 - 3 } & $\alpha=2$ & $\alpha=-2$ \\
\hline \hline 0 & 0 & 0 \\
\hline 0.1 & 3.0519 & 5.6988 \\
\hline 0.2 & 6.1039 & 11.3975 \\
\hline 0.3 & 9.1558 & 17.0963 \\
\hline 0.4 & 12.2077 & 22.7951 \\
\hline
\end{tabular}

The streamlines for the present problem are shown in Fig. 1b. Figs. 2 and 3 show that, for $\alpha>0$ or $\alpha<0$, the external magnetic field tends to decrease both the axial and radial velocities in the region in the middle of the two disks. Thus, for this region, the magnetic field acts like a drag force called the Lorentz force which decreases the fluid velocity. This results in generation of heat energy which remarkably increases the fluid temperature as shown in Figs. 2c and 3c. The influence of the nanoparticle volume fraction parameter on the velocity and temperature profiles is similar to that of the external magnetic field (Figs. 4 and 5) for the two cases of $\alpha$. The effect of $R$ on the velocity and temperature distribution is the same as that of $M$ for $\alpha>0$, whereas the opposite trend may be observed for $\alpha<0$ (Figs. 6 and 7). As the values 
of $\alpha$ are varied from negative to positive, the axial velocity component increases, whereas the radial velocity increases in the middle of the region between the disks and decreases near the disks (Figs. 8a and 8b). When the disks are approaching each other, an increase in the expansion ratio $\alpha$ results in increasing the temperature distribution across the disks. But in the other case, the temperature profiles rise only in the middle of the disks (Fig. 8c). Finally, Figs. 9a and 9b show that the viscous dissipation remarkably increases the temperature distribution across the disks, no matter if the disks are approaching or receding.

(a)

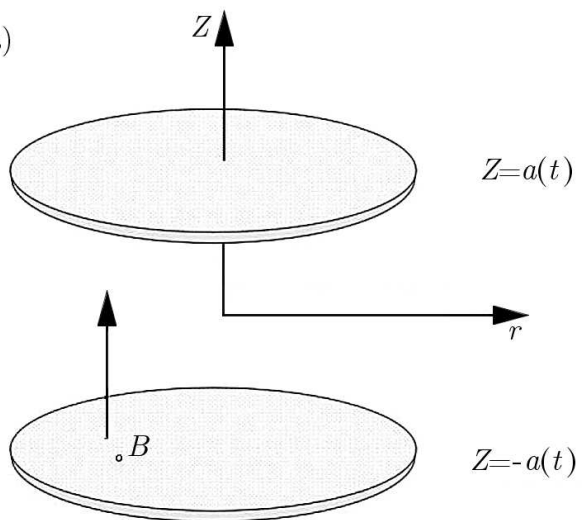

(b)

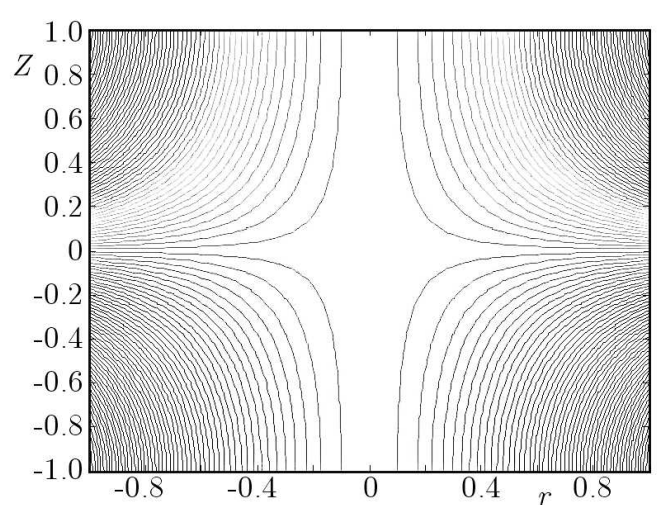

Fig. 1. (a) Physical model of the problem; (b) streamlines for the problem for $R=-10, \phi=0.3$, $\alpha=-5$ and $M=1$

(a)

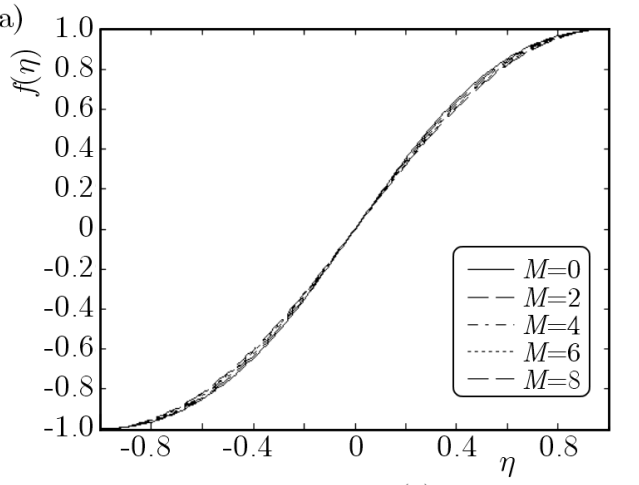

(b)

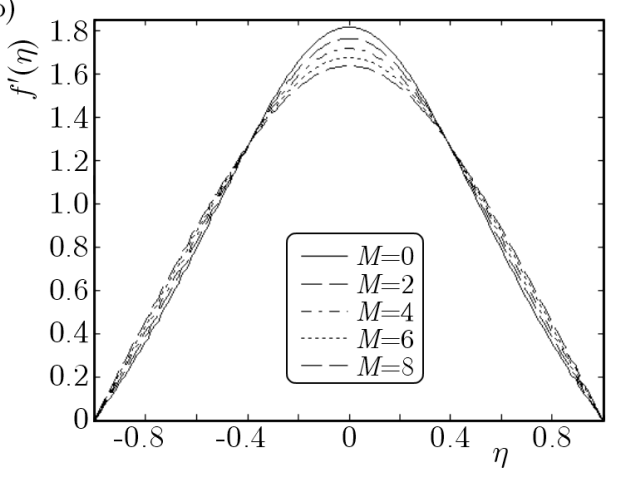

(c)

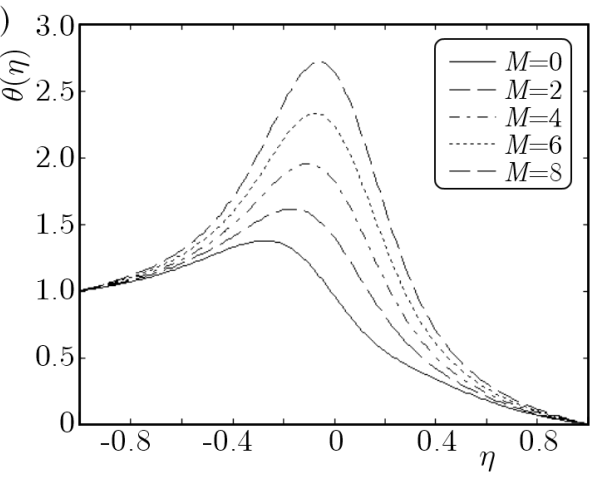

Fig. 2. (a) Axial velocity, (b) radial velocity and (c) temperature profiles for $R=-2, \phi=0.1, \alpha=2$ and $E c=0.1$ 
(a)

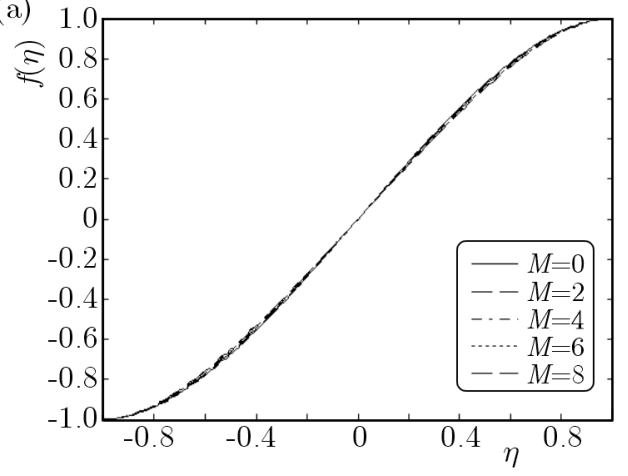

(b)

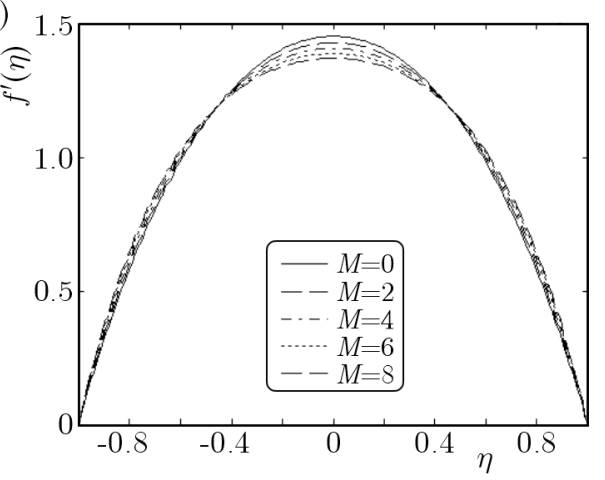

(c)

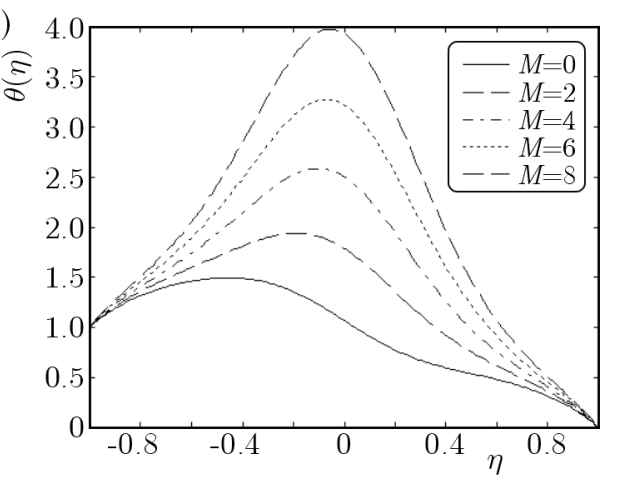

Fig. 3. (a) Axial velocity, (b) radial velocity and (c) temperature profiles for $R=-2, \phi=0.1, \alpha=-2$ and $E c=0.1$

(a)

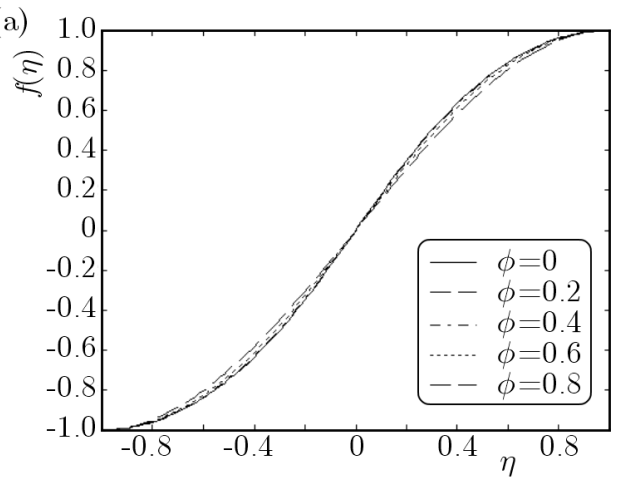

(b)

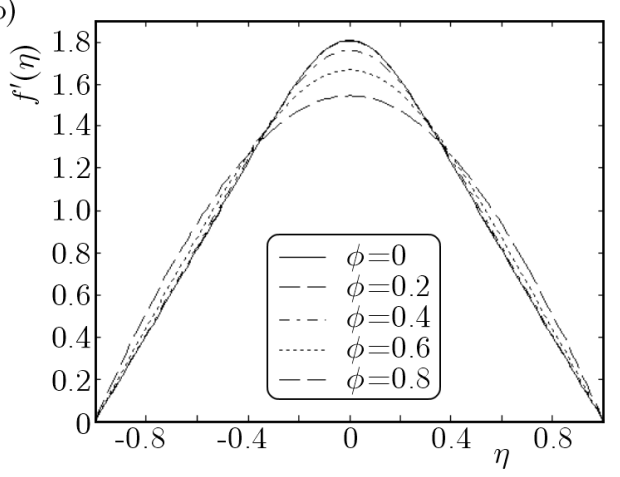

(c)

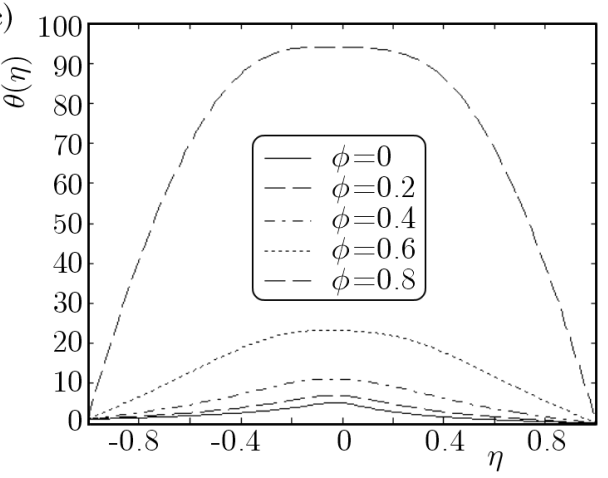

Fig. 4. (a) Axial velocity, (b) radial velocity and (c) temperature profiles for $M=1, \alpha=1, R=-10$ and $E c=0.1$ 
(a)

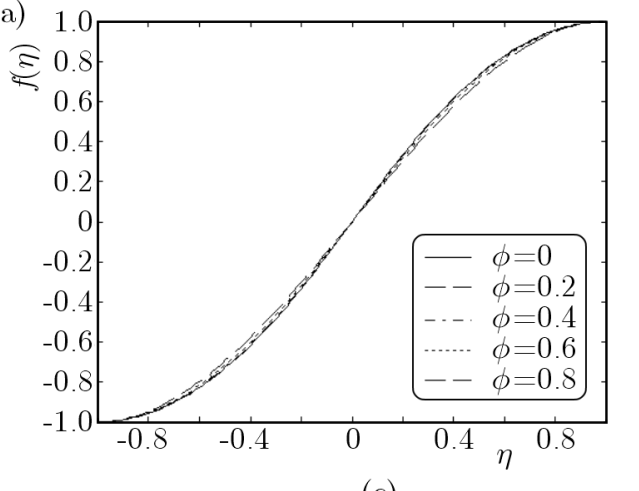

(b)

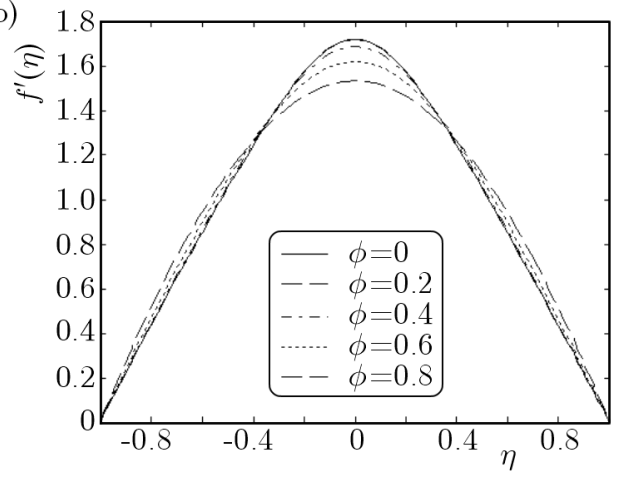

(c)

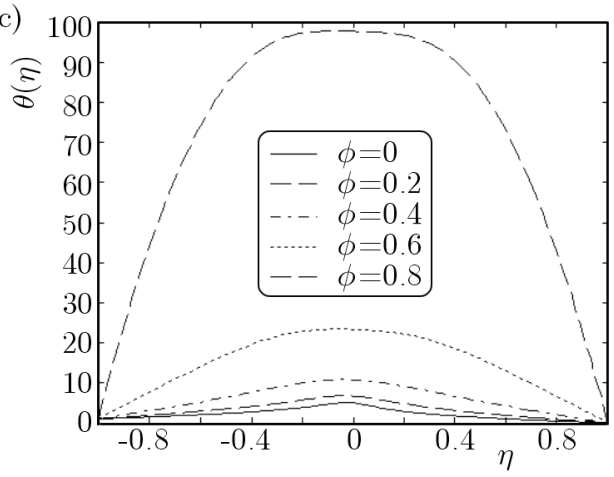

Fig. 5. (a) Axial velocity, (b) radial velocity and (c) temperature profiles for $M=1, \alpha=-1, R=-10$ and $E c=0.1$

(a)

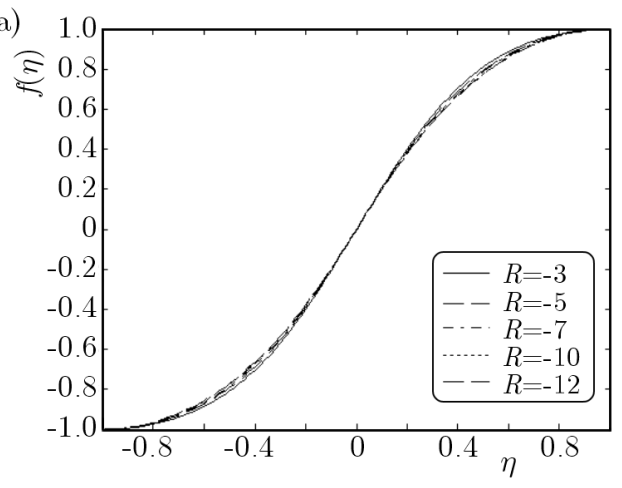

(b)

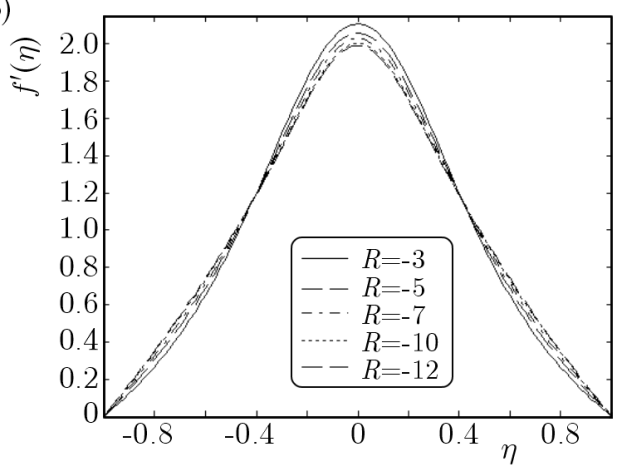

(c)

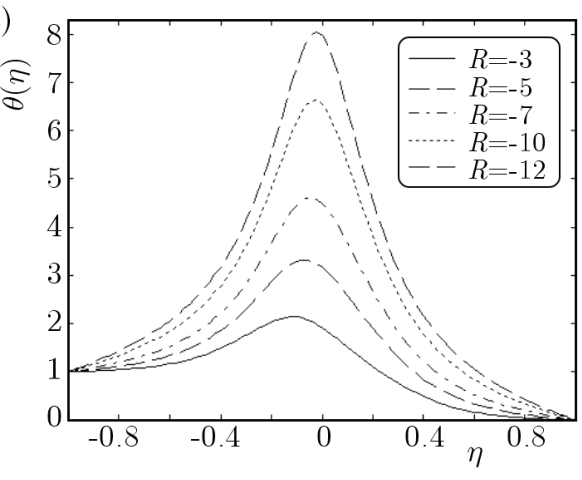

Fig. 6. (a) Axial velocity, (b) radial velocity and (c) temperature profiles for $M=1, \phi=0.1, \alpha=5$ and $E c=0.1$ 

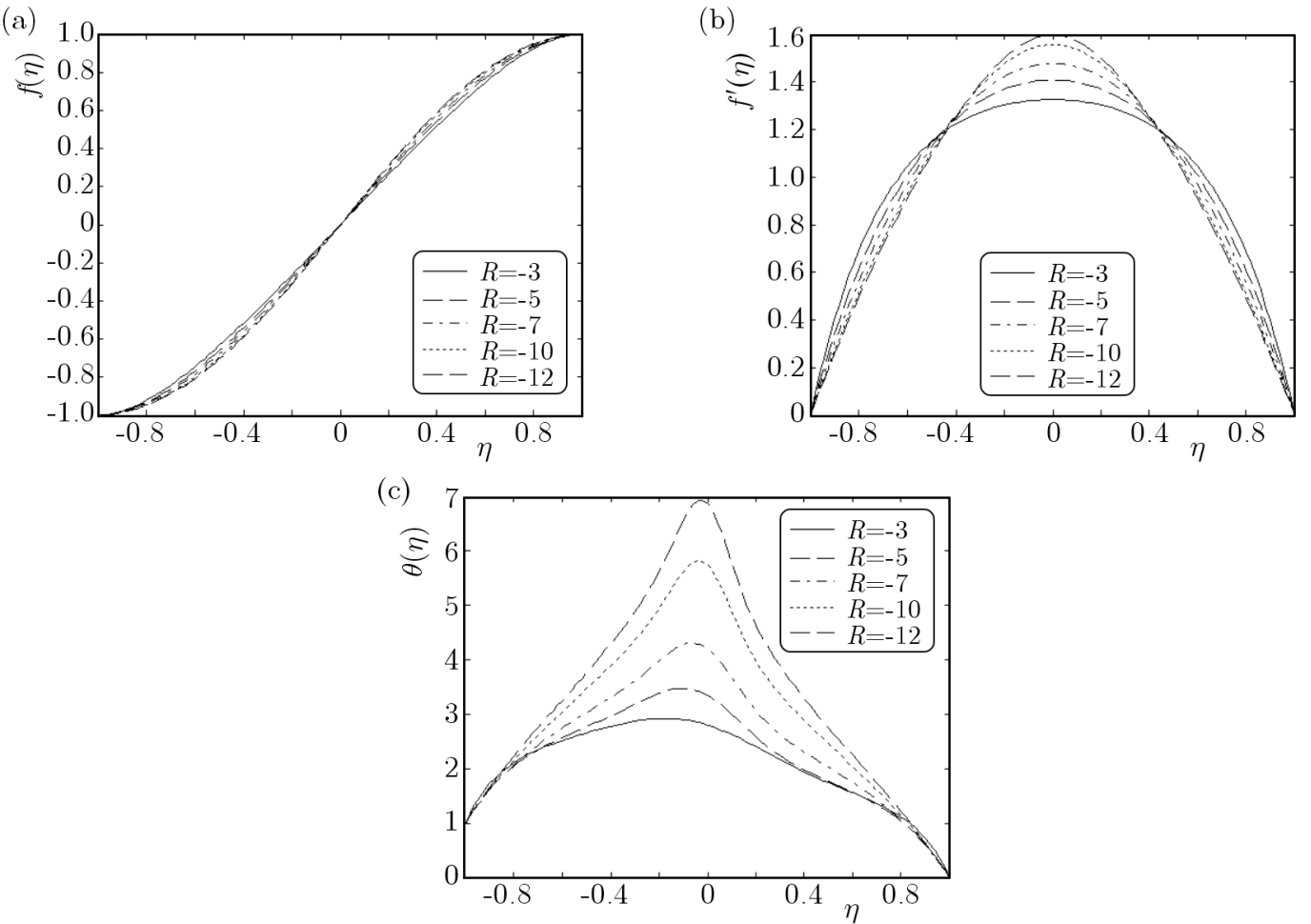

Fig. 7. (a) Axial velocity, (b) radial velocity and (c) temperature profiles for $M=1, \phi=0.1, \alpha=-5$ and $E c=0.1$
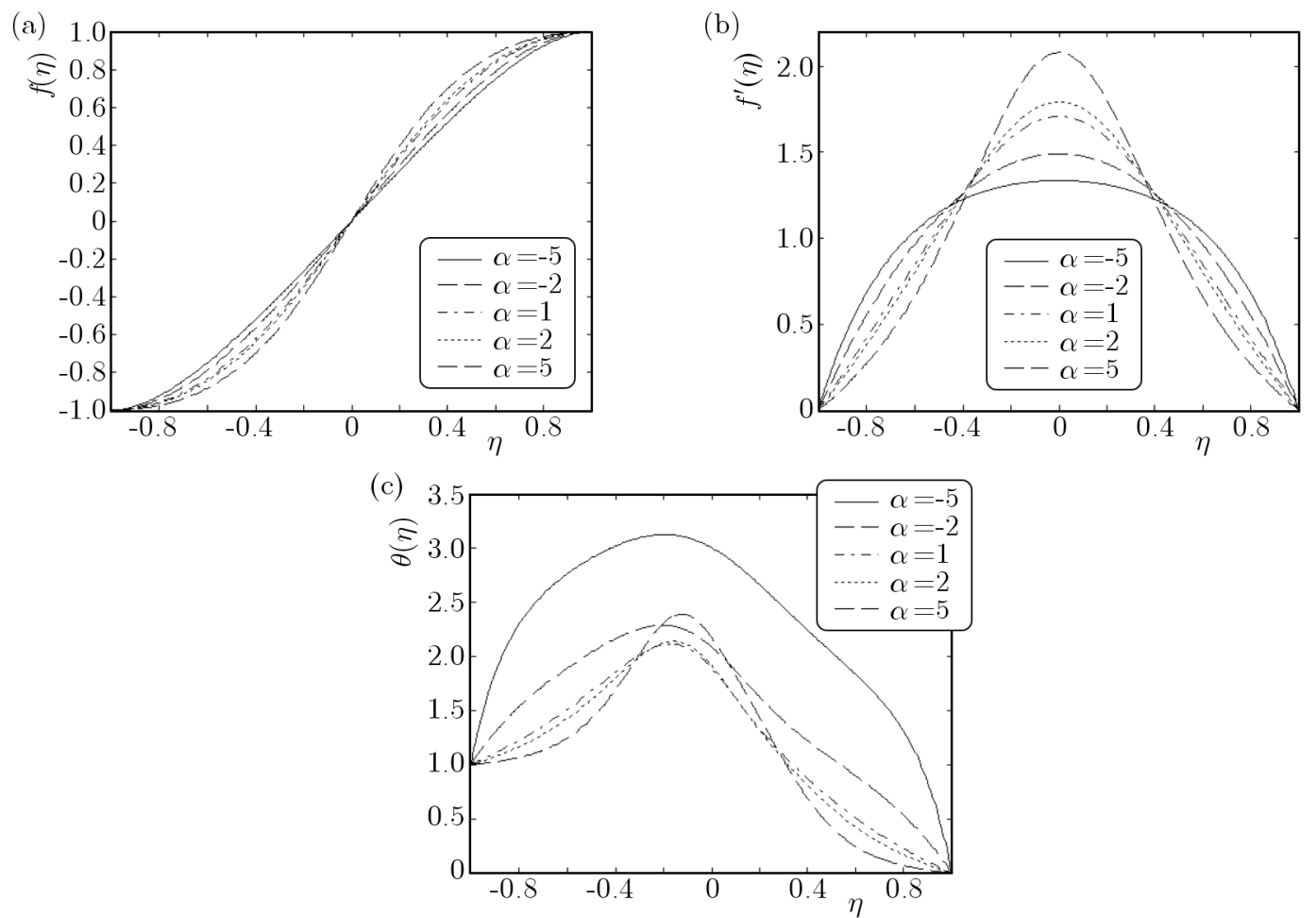

Fig. 8. (a) Axial velocity, (b) radial velocity and (c) temperature profiles for $M=1, \phi=0.2, R=-3$ and $E c=0.1$ 
(a)

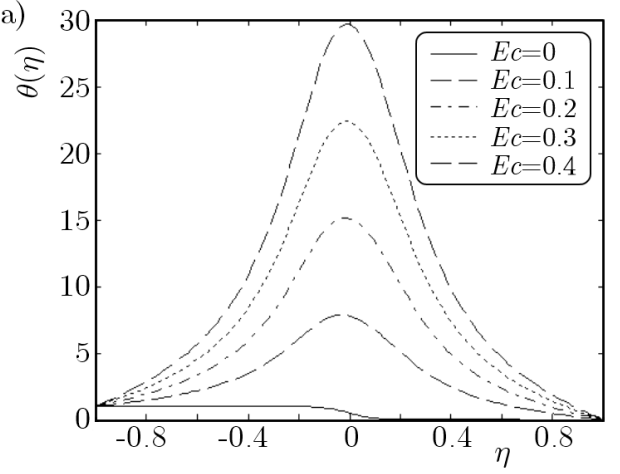

(b)

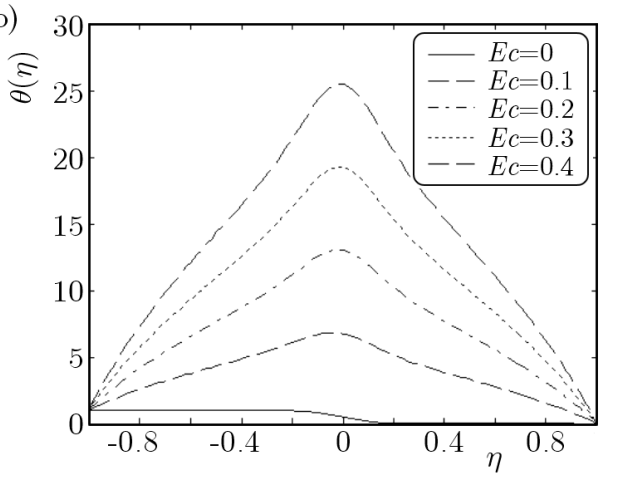

Fig. 9. Temperature profiles for: (a) $M=1, \alpha=5, R=-10$ and $\phi=0.2$, (b) $M=1, \alpha=-5$, $R=-10$ and $\phi=0.2$

\section{Conclusion}

In this paper, we have numerically studied how the governing parameters, namely the magnetic parameter $M$, the Reynolds number $R$, the nanoparticle volume fraction parameter $\phi$, the wall expansion ratio $\alpha$ and the Eckert number $E c$ affect the flow and heat transfer characteristics of the unsteady, laminar, incompressible and two-dimensional flow of a water-based nanofluid (containing the $\mathrm{Al}_{2} \mathrm{O}_{3}$ nanoparticles) between two orthogonally moving porous coaxial disks with suction.

When the disks are moving away $(\alpha>0)$ :

- shear stress at the disks increases with $M$ and $R$, whereas the opposite effect is observed for $\phi$ and $\alpha$,

- heat transfer rate at the disks increases with $M, R$ and $\phi$.

For the approaching disks $(\alpha<0)$ :

- shear stress at the disks increases with $M$ and $\alpha$, whereas the opposite effect is observed for $\phi$ and $R$,

- heat transfer rate at the disks increases with $M, R, \alpha$ and $\phi$.

All the governing parameters significantly increase the temperature distribution in the middle of the region between the approaching or receding disks. Finally, it is important to mention that the heat transfer characteristics of the nanofluids are not yet fully explored, and that is why complementary works are necessary to identify new and unique applications of these fluids.

\section{Acknowledgements}

The authors are extremely grateful to the Higher Education Commission of Pakistan for the financial support to carry out this research. The authors are also extremely grateful to the learned reviewer for useful comments improving quality of this paper.

\section{References}

1. Ali K., Ashraf M., Jameel N., 2013, Numerical simulation of MHD micropolar fluid flow and heat transfer in a channel with shrinking walls, Canadian Journal of Physics, 10.1139/cjp-2013-0324

2. Ashraf M., Kamal M.A., Syed K.S., 2009, Numerical simulation of a micropolar fluid between a porous disk and a non-porous disk, Journal of Applied Mathematics and Modelling, 33, 1933-1943

3. Ashraf M., Wehgal A.R., 2012, MHD flow and heat transfer of micropolar fluid between two porous disks, Applied Mathematics and Mechanics (English Edition), 33, 51-64 
4. BAnchok N., Ishak A., Pop I., 2011, Flow and heat transfer over a rotating porous disk in a nanofluid, Physica B, 406, 1767-1772

5. Buongiorno J., 2006, Convective transport in nanofluids, ASME Journal of Heat Transfer, 128, 240-250

6. Choi S.U.S., 1995, Enhancing thermal conductivity of fluids with nano particles, [In:] Developments and Applications of Non-Newtonian Flows, Siginer D.A., Wang H.P. (Eds.), 31, 99-105

7. Connor J.J., Boyd J., Avallone E.A., 1968, Standard Handbook of Lubrication Engineering, McGraw-Hill, New York

8. Das S.K., Choi S.U.S., 2007, Nanofluids: Science and Technology, Wiley, New Jersey

9. Deuflhard P., 1983, Order and step-size control in extrapolation methods, Numerical Mathematics, 41, 399-422

10. Elcrat A. R., 1976, On the radial flow of a viscous fluid between porous disks, Archive for Rational Mechanics and Analysis, 61, 91-96

11. Kakac S., Pramuanjaroenkij A., 2009, Review of convective heat transfer enhancement with nanofluids, International Journal of Heat and Mass Transfer, 52, 3187-3196

12. Majdalani J., Zhou C., Dawson C.A., 2002, Two dimensional viscous flows between slowly expanding or contracting walls with weak permeability, Journal of Biomechanics, 35, 1399-1403

13. Nakamura S., 1991, Applied Numerical Methods with Software, Prentice-Hall, 442-446

14. Rasmussen H., 1970, Steady viscous flow between two porous disks, Zeitschrift für Angewandte Mathematik und Physik, 21, 187-195

15. Roache P.J., Knupp P.M., 1993, Completed Richardson extrapolation, Communications in Numerical Methods in Engineering, 9, 365-374

16. Usha R., Ravindranthe R., 2001, Numerical study of film cooling on rotating disk, International Journal of Nonlinear Mechanics, 36, 147-154 